\title{
The distribution and impact of viral lineages in domains of life
}

\author{
Arshan Nasir ${ }^{1}$, Patrick Forterre ${ }^{2,3}$, Kyung Mo Kim ${ }^{4}$ and Gustavo Caetano-Anollés ${ }^{1 *}$ \\ ${ }^{1}$ Evolutionary Bioinformatics Laboratory, Department of Crop Sciences, Illinois Informatics Institute, University of Illinois, Urbana-Champaign, Urbana, IL, USA \\ 2 Unité BMGE, Institute Pasteur, Paris, France \\ ${ }^{3}$ Institut de Génétique and Microbiologie, Université Paris-Sud, CNRS UMR8621, Orsay, France \\ ${ }^{4}$ Microbial Resource Center, Korea Research Institute of Bioscience and Biotechnology, Daejeon, Korea \\ ${ }^{*}$ Correspondence: gca@illinois.edu
}

Edited and reviewed by:

Anton G. Kutikhin, Research Institute for Complex Issues of Cardiovascular Diseases under the Siberian Branch of the Russian Academy of Medical Sciences, Russia

Keywords: viruses, evolution, replicon, capsids, virion morphotype, diversity, domains of life

Living organisms can be conveniently classified into three domains, Archaea, Bacteria, and Eukarya (Woese et al., 1990). The three domains are united by several features that support the common origin of life including the presence of ribosomes, double-stranded DNA genomes, a nearly universal genetic code, physical compartments (i.e., membranes), and the ability to carry out metabolism and oxidationreduction reactions. In comparison, other types of genetic material and particles (e.g., viruses, plasmids, and other selfish genetic elements) are often excluded from the definition of "life" (for opposing views see Raoult and Forterre, 2008; Forterre, 2011, 2012a). However, they can still influence the evolution of cellular organisms, and in conjunction, establish complex life cycles.

Viruses impact our economy, medicine and agriculture due to their infectious nature. Viral infections transform the host cell into a virocell that no longer divides by binary fission but produces more viral particles or a ribovirocell in which the viral and cellular genomes coexist, the cell still dividing while producing virions (Forterre, 2011, 2012a). The virosphere (i.e., collection of all viruses) displays exceptional variability in virion morphologies and replication strategies. Viruses can be classified into DNA or RNA viruses, retroviruses or intermediate forms depending upon the type of replicon present inside the viral particle. Moreover, replicons could be linear, circular, singlestranded, double-stranded, or even segmented. The unprecedented diversity of replicon types has led to the proposal that viruses first invented DNA as means to trick the host defense systems (Forterre, 2002, 2005). Viruses can also transfer genes between species and enhance biodiversity (Nasir et al., 2012). Even more importantly, viruses appear to create massive amount of new genetic information, part of which can transfer to cells (Abroi and Gough, 2011; Forterre, 2011, 2012b). The discovery of "giant" viruses such as mimiviruses (La Scola et al., 2003), megaviruses (Arslan et al., 2011), pandoraviruses (Philippe et al., 2013), and pithoviruses (Legendre et al., 2014) now creates a continuum in genome size and functional complexity between the virosphere and cells. Still, viruses are neglected in phylogenetic studies because they lack a unifying genetic marker, similar to rRNA for cells, and because many biologists underestimate their genetic creativity. As a consequence, their role in the origin and evolution of modern life, and their impact on the ecology of our biosphere continue to be for the most part unrecognized (Koonin and Wolf, 2012). In this opinion article, we address the impact of viruses on the evolution of cells. We argue that viruses likely initiated major evolutionary shifts. Specifically, we consider that gain and loss of viral lineages often leads to divergent evolutionary trends even in closely related species. We emphasize that no evolutionary theory could be complete without accounting for the viral world and that viruses are responsible for ongoing adaptations in the cellular domains (see also Prangishvili et al., 2006; Forterre and Prangishvili, 2013; Koonin and Dolja, 2013).
The distribution of the association of viral replicon types with cells is extremely biased. For example, RNA viruses are completely absent in Archaea and are rare in Bacteria. In comparison, vertebrates host numerous RNA and retroviruses. Surprisingly, dsDNA viruses are rare in plants while dsRNA viruses are abundant in fungi. Similarly, retroviruses are integrated into the genomes of multicellular eukaryotes but are completely absent in the microbial genomes. In other words, specific relationships exist between the type of viral replicon and the host range. Viruses with a particular replicon may infect one group of organisms but may not replicate in another. Big jumps of viruses from one cellular lineage to another have been observed within the eukaryotic "division" such as animals (opisthokonts) and plants (viridiplantae), when a virus adapts to an established consortium of ecological partners. The same virus can sometimes infect both plant and animal cells when these are linked by their mode of life. One example is the Fiji disease virus (Reoviridae) that can replicate in both its insect vector (Delphacidae) and flowering plants (Kings et al., 2012). However, no modern virus is known to cross the barrier between domains. Therefore, while viruses may be able to jump hosts over short evolutionary time spans, crossing domain boundaries is less likely and not expected to compromise our inferences.

To obtain a quantitative view of viral diversity and its distribution among cellular domains, we extracted genome data from the Viral Genomes Resource at NCBI (Bao et al., 2004). This resource 
provides accurate, manually curated information about sequenced viral genomes that is minimally redundant. Generally, one sequenced genome portrays many isolates/strains of the same virus. Specifically, we investigated the host preferences for viruses with different replication strategies (Figure 1A) and contrasted virion morphologies (borrowed from ViralZone; Hulo et al., 2011) of virus families infecting different domain groups (Figure 1B). A birds-eye view of the distribution of viruses among hosts revealed that only 63 were exclusive to the archaeal domain (hereinafter referred to as archaeoviruses) (Figure 1A). In comparison, 1251 bacterial (bacterioviruses, formerly bacteriophages) and 2321 eukaryal viruses (eukaryoviruses) were identified. The low number of archaeoviruses is clearly due to a sampling bias (e.g., the low number of archaeal species screened for the presence of viral infection) since it has been shown that four different viruses can infect a single archaeal species (i.e., Aeropyrum pernix), each from a different family (Mochizuki et al., 2010, 2011, 2012). Despite their low number, archaeoviruses exhibit greater virion morphotype diversity compared to bacterioviruses [e.g., 4 unique virion morphotypes vs. none (Figure 1B); see also Pietilä et al., 2014]. In comparison, bacterial organisms host a vast number of described DNA viruses (1178 out of total 1760) but display very little family and morphotype diversity. In fact, $95 \%$ of the dsDNA bacterioviruses belong to just one order (Caudovirales) and only three families (Myoviridae, Podoviridae, and Siphoviridae). Moreover, only 9 virion morphologies have been observed in bacterioviruses (compared to 16 in archaeoviruses) (Pietilä et al., 2014). One explanation for the low diversity of bacterioviruses could be the invention of peptidoglycan-containing cell wall in Bacteria. The inability to traverse this barrier likely resulted in loss of many viral lineages in Bacteria (Forterre and Prangishvili, 2013; Prangishvili, 2013). Taken together, these observations suggest that Archaea are likely infected by a greater number of viral lineages than Bacteria. This is showcased by their virion morphologies diversity Figure 1) (Pina et al., 2011; Pietilä et al., 2014),

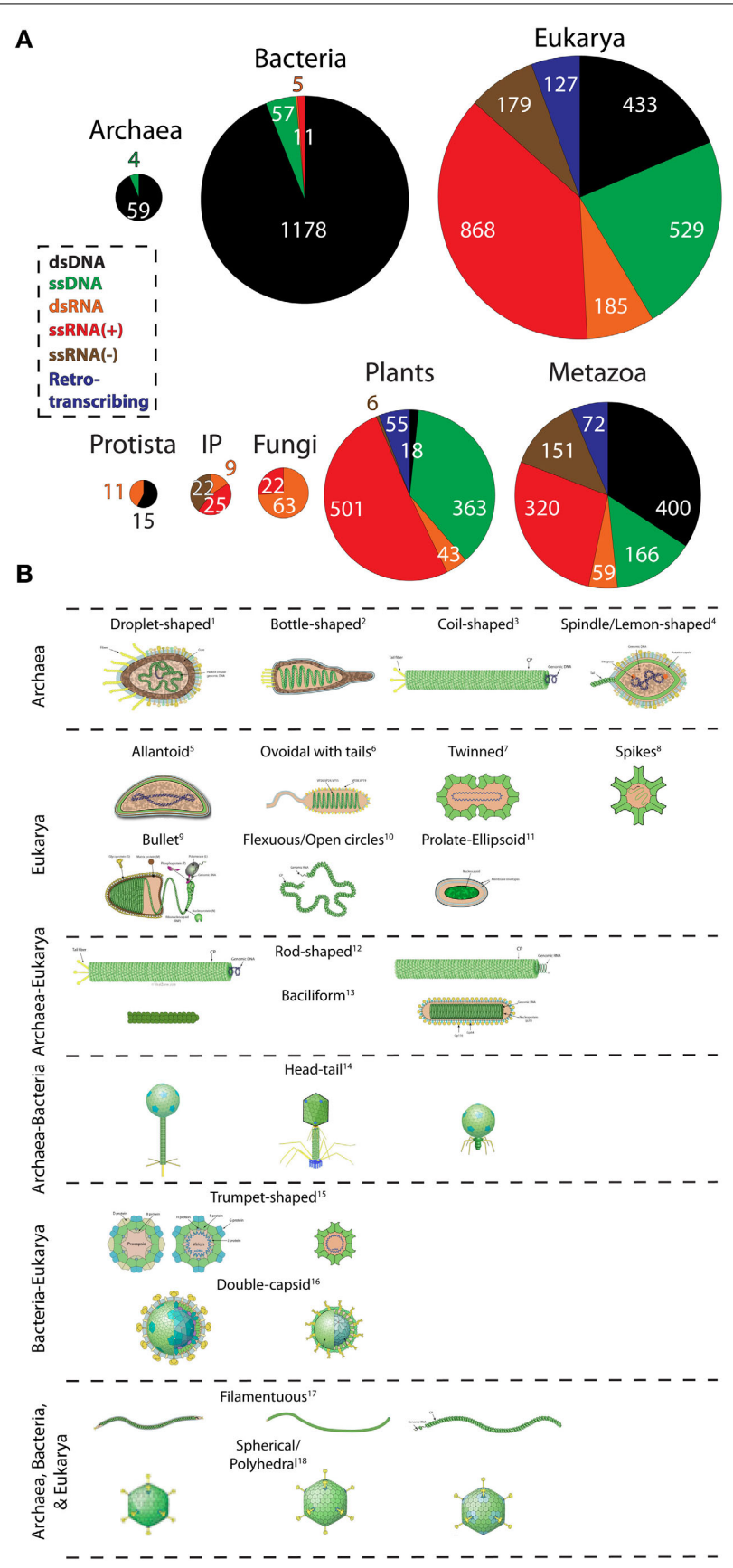

FIGURE 1 | The abundance and diversity of viral lineages in the domains of life. (A) Pie-charts describe the abundance of dsDNA, ssDNA, dsRNA, ssRNA(+), ssRNA(-), and retrotranscribing viruses in Archaea, Bacteria, and Eukarya, and within the major eukaryal divisions. Genome data from 3660 completely sequenced viral genomes corresponding to 1671 dsDNA, 610 ssDNA, 883 ssRNA(+), 179 ssRNA(-), 190 dsRNA, and 127 retrotranscribing viruses were retrieved from the Viral Genomes Resource (April, 2014). Additionally, two ssDNA archaeal viruses were identified from the literature (Pietilä et al., 2009; Mochizuki et al., 2012). Viruses that were unassigned to any order, genera, or species and unclassified viruses were excluded from sampling. Viruses were broadly classified according to host preferences into the following categories: Archaea, Bacteria, Protista (animal-like protists and brown algae), Invertebrates and plants (IP); Fungi (all fungi and fungi-like protists); Plants (all plants, green algae, and diatoms), and Metazoa (vertebrates, invertebrates, and human). Host information was available for roughly $99 \%$ (3633) of the sampled viruses. Pie-charts are proportional to the size of each distribution. (B) Virion morphotypes that are specific to a domain or are shared between domains are displayed. Virion pictures were borrowed 


\section{FIGURE 1 | Continued}

from the ViralZone web-resource (Hulo et al., 2011) and from Pietilä et al. (2014) and Pina et al. (2011). A keyword-based search was performed on text data to assign the most general morphotypes (e.g., rod-shaped, spherical, droplet-shaped, etc) to all viruses. More than one viridae with same morphotype is possible but not made explicit. The diagram does not always imply evolutionary relationship between viruses harboring common morphology. For example, archaeal and eukaryal rod-shaped viruses are probably not evolutionarily related (Goulet et al., 2009). Well-studied exceptions are head-tail caudovirales harboring the HK97 capsid fold and of polyhedral viruses harboring the double jelly roll fold (Abrescia et al., 2012). ${ }^{1}$ Guttaviridae; ${ }^{2}$ Ampullaviridae; ${ }^{3}$ Spiraviridae [name pending approval by ICTV]; ${ }^{4}$ Fuselloviridae; ${ }^{5}$ Ascoviridae; ${ }^{6}$ Nimaviridae; ${ }^{7}$ Geminiviridae; ${ }^{8}$ Astroviridae;

${ }^{9}$ Rhabdoviridae; ${ }^{10}$ Ophioviridae; ${ }^{11}$ Polydnaviridae; (left to right) ${ }^{12}$ Rudiviridae (Archaea); Virgaviridae (Eukarya); ${ }^{13}$ Clavaviridae (Archaea) Roniviridae (Eukarya); ${ }^{14}$ Siphoviridae, Myoviridae, and Podoviridae (Archaea and Bacteria); ${ }^{15}$ Microviridae (Bacteria), Circoviridae (Eukarya); ${ }^{16}$ Cystoviridae (Bacteria), Reoviridae (Eukarya); ${ }^{17}$ Lipothrixiviridae (Archaea), Inoviridae (Bacteria), Potyviridae (Eukarya);

${ }^{18}$ Sulfolobus turreted icosahedral virus (Archaea), Tectiviridae (Bacteria), Adenoviridae (Eukarya).

(which is expected to grow with improvements in our ability to isolate viruses from atypical habitats.

Interestingly, all archaeoviruses possess DNA replicons but no RNA genomes. The complete absence of RNA viruses in Archaea can be linked to high temperature RNA instability (Forterre, 2013). We speculate that escape from RNA viruses could be one major trigger for the evolution of modern Archaea (Forterre, 2013). Thus, loss of RNA viral lineages likely initiated archaeal migration to the harsh environments. One recent study reported the isolation of $\operatorname{ssRNA}(+)$ viruses from an archaea-rich community in a hot, acidic spring of Yellowstone National Park (Bolduc et al., 2012). However, their host tropism could not be established with confidence. Finally, four ssDNA viruses were recently isolated from Archaea (Pietilä et al., 2009; Mochizuki et al., 2012; Sencilo et al., 2012). Of these, Aeropyrum coil-shaped virus (Spiraviridae) is the largest known ssDNA virus and displays unique coil-shaped virion morphology (Figure 1B; Mochizuki et al., 2012).

Bacterioviruses are remarkably successful in Bacteria and are highly abundant. Their virions outnumber their bacterial hosts in oceans, balance microbial populations in the marine communities, and regulate biogeochemical cycles (Breitbart and Rohwer, 2005; Suttle, 2007; Rohwer and Thurber, 2009; Zhao et al., 2013). Among the dsDNA bacterioviruses, tailed-bacteriophages exhibit extensive similarities with archaeal caudovirales, suggesting that they form a monophyletic group (Krupovic et al., 2010). Archaeal and bacterial caudovirales have indeed been grouped in a single major evolutionary lineage, together with Herpesviridae. All of these viruses share the same Hong Kong fold (HK97) in their major capsid proteins and homologous packaging ATPases (Baker et al., 2005; Pell et al., 2009; Krupovic et al., 2010; Abrescia et al., 2012). Notably, it has been found recently that the capsid of Herpesviridae exhibits a small tail similar to those of Podoviridae (Schmid et al., 2012). These data suggest that viruses of the HK97-like lineage are very ancient and originated (most likely) prior to the last common ancestor of cells. Another example of viral lineage shared by the three domains is the so-called "PRD1/Adenovirus lineage" of dsDNA viruses characterized by a major capsid protein containing the doublejelly roll fold and a common packaging ATPase (Abrescia et al., 2012). In comparison, ssDNA bacterioviruses are not as successful in Bacteria and correspond to two major families, Inoviridae and Microviridae (smallest genomes among DNA viruses; Rosario et al., 2012). Viruses in this group replicate by converting their single-stranded DNA genome into a double-stranded intermediate form engineered by host polymerase. These viruses lack their own polymerase and share this property with the ssDNA viruses of Archaea and Eukarya.

In contrast to DNA viruses, RNA viruses are not as successful in Bacteria. Only, 5 dsRNA, and 11 ssRNA(+) bacterioviruses could be identified. In turn, none of the ssRNA(-) and retrotranscribing viruses associated with bacterial hosts. Among the RNA bacterioviruses,
dsRNA viruses (Cystoviridae) encode segmented genomes and infect mostly Pseudomonas species (Silander et al., 2005). Interestingly, Cystoviridae closely resembles eukaryal dsRNA viruses (i.e., Reoviridae and Totiviridae) in terms of life cycle and homologous RNA-dependentRNA-polymerase gene sequences (a virus hallmark) (Butcher et al., 1997). Unlike Archaea, Bacteria are also infected by ssRNA $(+)$ viruses (Leviviridae). These viruses are amongst the simplest and smallest known viruses, and historically yielded useful insights into mRNA function (Bollback and Huelsenbeck, 2001). Because RNA viruses (ssRNA and dsRNA) infect both Bacteria and Eukarya, their ancestors likely originated from a putative ancient world of cells with RNA genomes and RNA viruses (Forterre, 2005, 2006a,b). This points to the ancient existence of RNA viruses and suggests their loss from Archaea (since loss in one domain is more likely than the independent gain in two!). The instability of RNA at high temperatures supports this hypothesis, since it is likely that the last common ancestor of Archaea was a hyperthermophile (Brochier-Armanet et al., 2011).

Viruses with all possible types of replicons infect eukaryal organisms. RNA viruses are predominant and cover the entire taxonomic range within Eukarya (Figure 1A). Eukaryoviruses also exhibit many unique virion morphotypes not observed in the prokaryotic viruses and are unequally distributed in the major eukaryal groups (Figure 1). For example, dsDNA viruses are completely absent in fungi and are rare in plants (i.e., only found in green algae). This suggests that these groups have evolved sophisticated mechanisms to eliminate dsDNA viral infections. A good candidate is the cell wall structure found in plants, fungi, and algae. Differences in cell wall composition and rigidity greatly limit means of viral entry into the cell and serve as barriers to viral infections (Dimmock et al., 2007). However, loss of one viral lineage is apparently offset by the gain of other lineages. This is evident from the high RNA virus distribution among plants and fungi. The origin of the diversity and abundance of RNA viruses in eukaryotes but their near absence in prokaryotes is particularly puzzling (Koonin et al., 2006). 
For example, ssRNA(-) and retroviruses are highly successful in vertebrates. At first glance, it seems that organism complexity is proportional to the variety of viral infections. For instance, metazoa are infected by a host of retroviruses. Retroviruses can integrate their genomes into host DNA and thus alter gene expression patterns and trigger genomic rearrangements (Arkhipova et al., 2012). These activities can lead to production of novel genes and advanced machineries (Forterre, 2013). In fact, telomerase enzymes are homologous to retroviral proteins and neocentromeres are formed by epigenetic regulation of transposable elements (Singer, 1995; Chueh et al., 2009), both likely transferred from viruses to host cells much earlier in evolution. This argument is further supported by the absence of RNA and retroviruses from unicellular eukaryotes such as yeast, which resemble a prokaryotic lifestyle (Forterre, 2013). Thus, co-evolution between viruses and their hosts may have led to organism complexity in the eukaryotic domain.

The diversity of eukaryoviruses is intriguing, both in terms of genome structure and virion morphology (see Figure 1B). In particular, retrotranscribing, ssRNA $(-)$, and many DNA virus families are only present in eukaryotes. Surprisingly, although Archaea and eukaryotes are very similar in term of their basic molecular biology, there are no viral lineages specific for these two domains (Forterre, 2013). Virions with rod-shaped morphology are up to now specific for Archaea and Eukarya (Figure 1B), but they harbor DNA and RNA genomes, respectively, and it is unclear if their major coat proteins are evolutionary related (Goulet et al., 2009). The same is probably also true for bacilliform viruses. Notably, the diversity and specificity of eukaryoviruses is difficult to reconcile with the archaeon-bacterium fusion scenarios for the origin of eukaryotes (e.g., Martin and Müller, 1998), as recently argued (Forterre, 2013).

To conclude, the distribution of viral lineages follows an ancient, highly dynamic and ongoing process that impacts the evolution of organisms. New viral lineages often arise from existing ones and may cross species barriers to infect new hosts (e.g., parvovirus; Shackelton et al., 2005), putting enormous evolutionary pressure on cellular organisms and prompting them to unfold molecular and cellular innovation (Forterre and Prangishvili, 2009) in the search of either simplicity or complexity.

\section{ACKNOWLEDGMENTS}

Arshan Nasir is recipient of a Chateaubriand fellowship. Research has been supported by grants from the National Science Foundation (OISE-1132791) and the United States Department of Agriculture (ILLU-802-909 and ILLU-483-625) to Gustavo CaetanoAnollés, from the European Research Council to Patrick Forterre under the European Union's Seventh Framework Programme (FP/2007-2013)/Project EVOMOBIL_ERC Grant Agreement no. 340440, and from the KRIBB Research Initiative Program and the Next-Generation BioGreen 21 Program, Rural Development Administration (PJ0090192014) to Kyung Mo Kim.

\section{REFERENCES}

Abrescia, N. G. L., Bamford, D. H., Grimes, J. M., and Stuart, D. I. (2012). Structure unifies the viral universe. Аnnu. Rev. Biochem. 81, 795-822. doi: 10.1146/annurev-biochem-060910-095130

Abroi, A., and Gough, J. (2011). Are viruses a source of new protein folds for organisms? - Virosphere structure space and evolution. Bioessays 33, 626-635. doi: 10.1002/bies.201000126

Arkhipova, I. R., Batzer, M. A., Brosius, J., Feschotte, C., Moran, J. V., Schmitz, J., et al. (2012). Genomic impact of eukaryotic transposable elements. Mob. DNA 3, 19. doi: 10.1186/1759-8753-3-19

Arslan, D., Legendre, M., Seltzer, V., Abergel, C., and Claverie, J. M. (2011). Distant Mimivirus relative with a larger genome highlights the fundamental features of Megaviridae. Proc. Natl. Acad. Sci. U.S.A. 108, 17486-17491. doi: 10.1073/pnas.1110889108

Baker, M. L., Jiang, W., Rixon, F. J., and Chiu, W. (2005). Common ancestry of herpesviruses and tailed DNA bacteriophages. J. Virol. 79, 14967-14970. doi: 10.1128/JVI.79.23.14967-14970.2005

Bao, Y., Federhen, S., Leipe, D., Pham, V., Resenchuk, S., Rozanov, M., et al. (2004). National center for biotechnology information viral genomes project. J. Virol. 78, 7291-7298. doi: 10.1128/JVI.78.14.7291-7298.2004

Bolduc, B., Shaughnessy, D. P., Wolf, Y. I., Koonin, E. V., Roberto, F. F., and Young, M. (2012). Identification of novel positive-strand RNA viruses by metagenomic analysis of archaea-dominated Yellowstone hot springs. J. Virol. 86, 5562-5573. doi: 10.1128/JVI.07196-11

Bollback, J. P., and Huelsenbeck, J. P. (2001). Phylogeny, genome evolution, and host specificity of single-stranded RNA bacteriophage (family Leviviridae). J. Mol. Evol. 52, 117-128. doi: 10.1007/s002390010140

Breitbart, M., and Rohwer, F. (2005). Here a virus, there a virus, everywhere the same virus? Trends Microbiol. 13, 278-284. doi: 10.1016/j.tim.2005.04.003

Brochier-Armanet, C., Forterre, P., and Gribaldo, S. (2011). Phylogeny and evolution of the Archaea: one hundred genomes later. Curr. Opin. Microbiol. 14, 274-281. doi: 10.1016/j.mib.2011. 04.015

Butcher, S., Dokland, T., Ojala, P., Bamford, D., and Fuller, S. (1997). Intermediates in the assembly pathway of the double-stranded RNA virus Phi6. EMBO J. 16, 4477-4487. doi: 10.1093/emboj/16.14.4477

Chueh, A. C., Northrop, E. L., Brettingham-Moore, K. H., Choo, K. A., and Wong, L. H. (2009). LINE retrotransposon RNA is an essential structural and functional epigenetic component of a core neocentromeric chromatin. PLoS Genet. 5:e1000354. doi: 10.1371/journal.pgen.1000354

Dimmock, N. J., Easton, A. J., and Leppard, K. N. (2007). Introduction to Modern Virology, 6th Edn. Malden, MA: Blackwell Publishing Limited.

Forterre, P. (2002). The origin of DNA genomes and DNA replication proteins. Curr. Opin. Microbiol. 5, 525-532. doi: 10.1016/S1369-5274(02)00360-0

Forterre, P. (2005). The two ages of the RNA world, and the transition to the DNA world: a story of viruses and cells. Biochimie 87, 793-803. doi: 10.1016/j.biochi.2005.03.015

Forterre, P. (2006a). Three RNA cells for ribosomal lineages and three DNA viruses to replicate their genomes: a hypothesis for the origin of cellular domain. Proc. Natl. Acad. Sci. U.S.A. 103, 3669-3674. doi: 10.1073/pnas.0510333103

Forterre, P. (2006b). The origin of viruses and their possible roles in major evolutionary transitions. Virus Res. 117, 5-16. doi: 10.1016/j.virusres.2006.01.010

Forterre, P. (2011). Manipulation of cellular syntheses and the nature of viruses: the virocell concept. Comptes Rendus Chimie 14, 392-399. doi: 10.1016/j.crci.2010.06.007

Forterre, P. (2012a). Virocell Concept, The. eLS Chichester, West Sussex: John Wiley \& Sons, Ltd. doi: 10.1002/9780470015902.a0023264

Forterre, P. (2012b). Darwin's goldmine is still open: variation and selection run the world. Front. Cell. Infect. Microbiol. 2:106. doi: 10.3389/fcimb.2012.00106

Forterre, P. (2013). The common ancestor of Archaea and Eukarya was not an archaeon. Archaea 2013, 372396. doi: 10.1155/2013/372396

Forterre, P., and Prangishvili, D. (2009). The great billion-year war between ribosome- and capsidencoding organisms (cells and viruses) as the major source of evolutionary novelties. Ann. N. Y. Acad. Sci. 1178, 65-77. doi: 10.1111/j.17496632.2009.04993.x

Forterre, P., and Prangishvili, D. (2013). The major role of viruses in cellular evolution: facts and hypotheses. Curr. Opin. Virol. 3, 558-565. doi: 10.1016/j.coviro.2013.06.013

Goulet, A., Blangy, S., Redder, P., Prangishvili, D., Felisberto-Rodrigues, C., Forterre, P., et al. (2009). Acidianus filamentous virus 1 coat proteins 
display a helical fold spanning the filamentous archaeal viruses lineage. Proc. Natl. Acad. Sci. U.S.A. 106, 21155-21160. doi: 10.1073/pnas.0909 893106

Hulo, C., de Castro, E., Masson, P., Bougueleret, L., Bairoch, A., Xenarios, I., et al. (2011). ViralZone: a knowledge resource to understand virus diversity. Nucleic Acids Res. 39, D576-D582. doi: 10.1093/nar/gkq901

Kings, A. M. Q., Adams, M. J., Carstens, E. B., and Lefkowitz, E. J. (2012). Virus Taxonomy: Classification and Nomenclature of Viruses: Ninth Report of the International Committee on Taxonomy of Viruses. San Diego, CA: Elsevier Academic Press.

Koonin, E. V., and Dolja, V. V. (2013). A virocentric perspective on the evolution of life. Curr. Opin. Virol. 3, 546-557. doi: 10.1016/j.coviro.2013.06.008

Koonin, E. V., Senkevich, T. G., and Dolja, V. V. (2006). The ancient Virus World and evolution of cells. Biol. Direct 1, 29. doi: 10.1186/17456150-1-29

Koonin, E. V., and Wolf, Y. I. (2012). Evolution of microbes and viruses: a paradigm shift in evolutionary biology? Front. Cell. Infect. Microbiol. 2:119. doi: $10.3389 /$ fcimb.2012.00119

Krupovic, M., Forterre, P., and Bamford, D. H. (2010). Comparative analysis of the mosaic genomes of tailed archaeal viruses and proviruses suggests common themes for virion architecture and assembly with tailed viruses of bacteria. J. Mol. Biol. 397, 144-160. doi: 10.1016/j.jmb.2010. 01.037

La Scola, B., Audic, S., Robert, C., Jungang, L., de Lamballerie, X., Drancourt, M., et al. (2003). A giant virus in amoebae. Science 299, 2033. doi: $10.1126 /$ science. 1081867

Legendre, M., Bartoli, J., Shmakova, L., Jeudy, S., Labadie, K., Adrait, A., et al. (2014). Thirtythousand-year-old distant relative of giant icosahedral DNA viruses with a pandoravirus morphology. Proc. Natl. Acad. Sci. U.S.A. 111, 4274-4279. doi: 10.1073/pnas.1320670111

Martin, M., and Müller, M. (1998). The hydrogen hypothesis for the first eukaryote. Nature 392, 37-41. doi: 10.1038/32096

Mochizuki, T., Krupovic, M., Pehau-Arnaudet, G., Sako, Y., Forterre, P., and Prangishvili, D. (2012). Archaeal virus with exceptional virion architecture and the largest single-stranded DNA genome. Proc. Natl. Acad. Sci. U.S.A. 109, 13386-13391. doi: 10.1073/pnas.1203668109

Mochizuki, T., Sako, Y., and Prangishvili, D. (2011). Provirus induction in hyperthermophilic archaea: characterization of Aeropyrum pernix spindleshaped Virus 1 and Aeropyrum pernix Ovoid Virus 1. J. Bacteriol. 193, 5412-5419. doi: 10.1128/JB.05101-11
Mochizuki, T., Yoshida, T., Tanaka, R., Forterre, P., Sako, Y., and Prangishvili, D. (2010). Diversity of viruses of the hyperthermophilic archaeal genus Aeropyrum, and isolation of the Aeropyrum pernix bacilliform virus 1, APBV1, the first representative of the family Clavaviridae. Virology 402, 347-354. doi: 10.1016/j.virol.2010.03.046

Nasir, A., Kim, K. M., and Caetano-Anolles, G. (2012). Giant viruses coexisted with the cellular ancestors and represent a distinct supergroup along with superkingdoms Archaea, Bacteria and Eukarya. BMC Evol. Biol. 12:156. doi: 10.1186/1471-214812-156

Pell, L. G., Kanelis, V., Donaldson, L. W., Howell, P. L., and Davidson, A. R. (2009). The phage lambda major tail protein structure reveals a common evolution for long-tailed phages and the type VI bacterial secretion system. Proc. Natl. Acad. Sci. U.S.A. 106, 4160-4165. doi: 10.1073/pnas.0900 044106

Philippe, N., Legendre, M., Doutre, G., Couté, Y., Poirot, O., Lescot, M., et al. (2013). Pandoraviruses: amoeba viruses with genomes up to $2.5 \mathrm{Mb}$ reaching that of parasitic eukaryotes. Science 341, 281-286. doi: $10.1126 /$ science. 1239181

Pietilä, M. K., Demina, T. A., Atanasova, N. S., Oksanen, J. M., and Bamford, D. H. (2014). Archaeal viruses and bacteriophages: comparisons and contrasts. Trends Microbiol. doi: 10.1016/j.tim.2014.02.007. [Epub ahead of print].

Pietilä, M. K., Roine, E., Paulin, L., Kalkkinen, N., and Bamford, D. H. (2009). An ssDNA virus infecting archaea: a new lineage of viruses with a membrane envelope. Mol. Microbiol. 72, 307-319. doi: 10.1111/j.1365-2958.2009.06642.x

Pina, P., Bize, A., Forterre, P., and Prangishvili, D. (2011). The archeoviruses. FEMS Microbiol. Rev. 35, 1035-1054. doi: 10.1111/j.1574-6976.2011.00280.x

Prangishvili, D. (2013). The wonderful world of archaeal viruses. Annu. Rev. Microbiol. 67, 565-585. doi: 10.1146/annurev-micro-092412155633

Prangishvili, D., Forterre, P., and Garrett, R. A. (2006). Viruses of the Archaea: a unifying view. Nat. Rev. Microbiol. 4, 837-848. doi: 10.1038/ nrmicro1527

Raoult, D., and Forterre, P. (2008). Redefining viruses: lessons from mimivirus. Nat. Rev. Microbiol. 6, 315-319. doi: 10.1038/nrmicro1858

Rohwer, F., and Thurber, R. V. (2009). Viruses manipulate the marine environment. Nature 459, 207-212. doi: 10.1038/nature08060

Rosario, K., Dayaram, A., Marinov, M., Ware, J., Kraberger, S., Stainton, D., et al. (2012). Diverse circular ssDNA viruses discovered in dragonflies (Odonata: Epiprocta). J. Gen. Virol. 93, 2668-2681. doi: 10.1099/vir.0.045948-0
Schmid, M. F., Hecksel, C. W., Rochat, R. H., Bhella, D., Chiu, W., and Rixon, F. J. (2012). A tail-like assembly at the portal vertex in intact herpes simplex type-1 virions. PLoS Pathog. 8:e1002961. doi: 10.1371/journal.ppat.1002961

Sencilo, A., Paulin, L., Kellner, S., Helm, M., and Roine, E. (2012). Related haloarchaeal pleomorphic viruses contain different genome types. Nucleic Acids Res. 40, 5523-5534. doi: 10.1093/nar/gks215

Shackelton, L. A., Parrish, C. R., Truyen, U., and Holmes, E. C. (2005). High rate of viral evolution associated with the emergence of carnivore parvovirus. Proc. Natl. Acad. Sci. U.S.A. 102, 379-384. doi: 10.1073/pnas.0406765102

Silander, O. K., Weinreich, D. M., Wright, K. M., O'Keefe, K. J., Rang, C. U., Turner, P. E., et al. (2005). Widespread genetic exchange among terrestrial bacteriophages. Proc. Natl. Acad. Sci. U.S.A. 102, 19009-19014. doi: 10.1073/pnas.0503074102

Singer, M. F. (1995). Unusual reverse transcriptases. J. Biol. Chem. 270, 24623-24626. doi: 10.1074/jbc.270.42.24623

Suttle, C. A. (2007). Marine viruses - major players in the global ecosystem. Nat. Rev. Microbiol. 5, 801-812. doi: 10.1038/nrmicro1750

Woese, C. R., Kandler, O., and Wheelis, M. L. (1990). Towards a natural system of organisms: proposal for the domains Archaea, Bacteria, and Eucarya. Proc. Natl. Acad. Sci. U.S.A. 87, 4576-4579. doi: 10.1073/pnas.87.12.4576

Zhao, Y., Temperton, B., Thrash, J. C., Schwalbach, M. S., Vergin, K. L., Landry, Z. C., et al. (2013). Abundant SAR11 viruses in the ocean. Nature 494, 357-360. doi: 10.1038/nature11921

Conflict of Interest Statement: The authors declare that the research was conducted in the absence of any commercial or financial relationships that could be construed as a potential conflict of interest.

Received: 10 April 2014; accepted: 11 April 2014; published online: 30 April 2014.

Citation: Nasir A, Forterre P, Kim KM and CaetanoAnollés $G$ (2014) The distribution and impact of viral lineages in domains of life. Front. Microbiol. 5:194. doi: 10.3389/fmicb.2014.00194

This article was submitted to Evolutionary and Genomic Microbiology, a section of the journal Frontiers in Microbiology.

Copyright (c) 2014 Nasir, Forterre, Kim and CaetanoAnollés. This is an open-access article distributed under the terms of the Creative Commons Attribution License (CC BY). The use, distribution or reproduction in other forums is permitted, provided the original author(s) or licensor are credited and that the original publication in this journal is cited, in accordance with accepted academic practice. No use, distribution or reproduction is permitted which does not comply with these terms. 